\title{
ML and MMSE Equalizers in Frequency selective and Flat fading Channels
}

\author{
Amit Grover \\ SBSSTC, Ferozepur \\ Punjab, India. \\ E-mail:amitgrover321@gmail.com
}

\begin{abstract}
One of the techniques to achieve the different challenges in wireless communication systems is MIMO systems which ensure a wide coverage, high throughput and reliable services by enabling spatial dimension and by considering multiple numbers of transmitter and receiver antennas. In this article, the performance of ML and MMSE equalizers has been compared in terms of BER in Frequency selective and flat fading channels. The performance of MMSE is worst in frequency selective channel as compared to others. It has been concluded that the performance of ML is better among these two equalizers, specifically in case of Binary Phase shift Keying.
\end{abstract}

Keywords - component- Quadrature Phase Shift Key (QPSK), Binary Phase Shift Key (BPSK), Minimum mean-squared error (MMSE), Maximum likelihood (ML), Bit error rate (BER).

\section{INTRODUCTION}

MIMO offers significant increases in data throughput and link range without additional bandwidth or transmit power because of the features of MIMO systems [5]. Communication in wireless channels is impaired predominantly by multipath fading. The Symbol mapper mapped the interleaved codeword's to data symbols and then these data symbols are applied as input to the Space Time-encoder which are again mapped to transmit antennas by space-time pre-coding block and are received at the antenna array by passing through the channel.

\section{MIMO SYSTEM MODEL}

By considering a MIMO system with a transmit array of $\mathrm{M}_{\mathrm{T}}$ antennas and a receive array of $\mathrm{M}_{\mathrm{R}}$ antennas [11], we can represent the MIMO channel [13] at a given time instant as a matrix.

\section{MIMO CHANNEL}

By considering an extreme condition where all antenna elements at the transmitter are collocated and likewise at the receiver, the de-correlation between the channel elements will increase with antenna spacing. In the presence of an LOS component between the transmitter and the receiver, the MIMO channel may be modeled as the sum of a fixed component and a fading component.

$$
\mathrm{H}=\sqrt{\frac{k}{1+k}} \boldsymbol{H}+\sqrt{\frac{k}{1+k}} H_{W}
$$

So the appropriate knowledge of the channel is required.

\section{EQUALIZATION TECHNIQUES}

\section{A. Minimum mean square error (MMSE)}

The MMSE detector is the optimal detection that seeks to balance between cancelation of the interference and reduction of noise enhancement [13].

\section{B. Maximum Likelihood (ML)}

Maximum likelihood detection [13] calculates the Euclidean distance between received signal vector and the product of all possible transmitted signal vectors with the given channel $\mathrm{H}$, and finds the one with minimum distance.

\section{SIMULATION AND RESULTS}

Using MATLAB, the different simulation results are shown in the different graphs, which provide the comparison of the BER for different modulation techniques using different equalizers like MMSE and ML with Flat fading and frequency selective channels.

Fig.1 (a) and Fig. 1(b) shows the comparison of BER for different modulations with MMSE equalizer in Rayleigh and Rician Flat Fading channels.

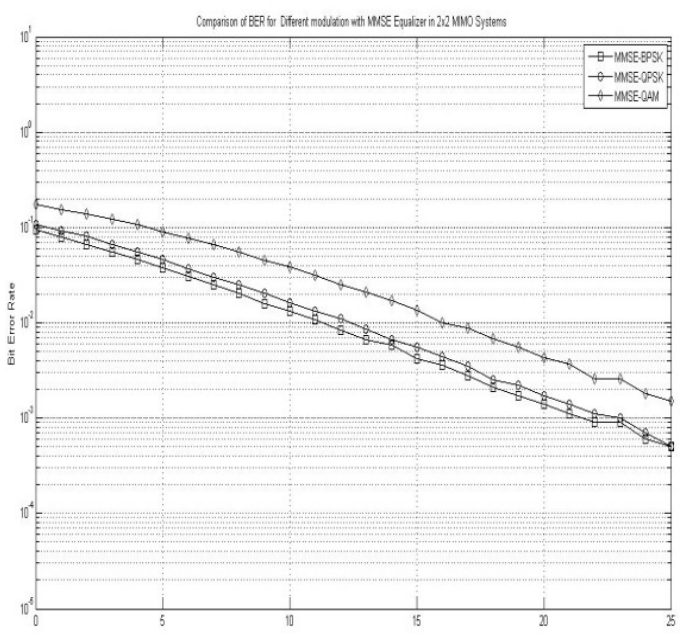

Fig.1 (a) 


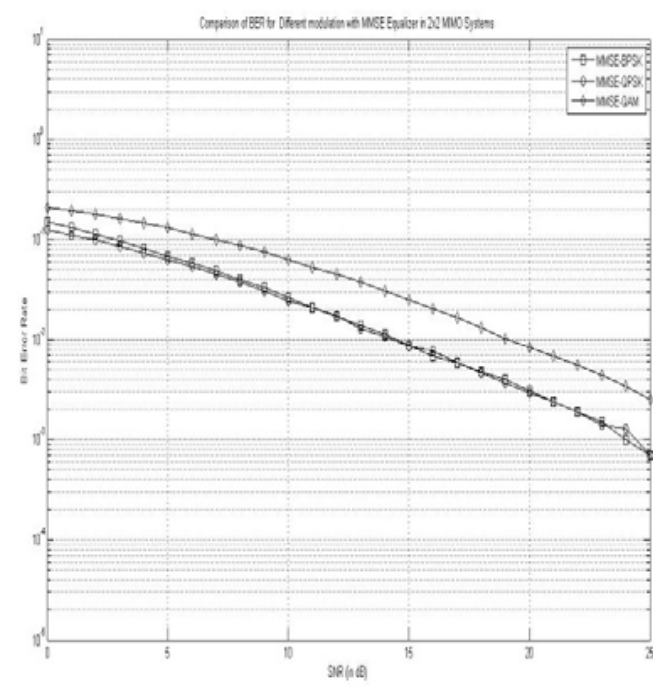

Fig.1 (b)

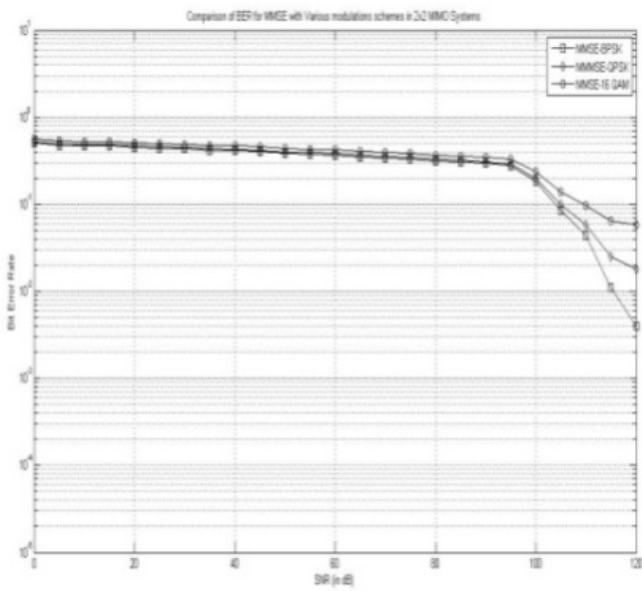

Fig.1(c)

"Fig.1"shows the comparison of BER for different modulations with MMSE equalizer

It has been observed from the figure. 1(a) that in Rayleigh Flat Fading Channel with the BPSK modulation we get the best result in comparison to 16 QAM whereas the result from BPSK and QPSK are almost same. So the order of performance in decreasing order is MMSE-BPSK > MMSE-QPSK > MMSE-16 QAM. MMSE is one of the simplest equalizer to simulate and also less complex. In case of Rican Flat Fading Channel, we can analyze from Figure 1(b) the simulated values for BER at $5 \mathrm{~dB}$ SNR are, for BPSK 0.0546, for QPSK 0.0633 and for 16 QAM 0.1308 . Thus, BPSK modulation gives us better result than QPSK and 16QAM, but the result with QPSK and BPSK are almost same. The MMSE equalizer doesn't work well in
Frequency selective fading channel. As it is clear from the figure 1(c), BER remains constant for a large SNR and we get a deviation in BER at large SNR. When we simulated MMSE equalizer with BPSK QPSK and 16 QAM by using MIMO 2 x2 Frequency selective fading channel, At $50 \mathrm{~dB}$ SNR, the BER for BPSK is 0.3850 , BER for QPSK is 0.3991 and BER for 16 QAM is 0.4390.Thus the performance of BPSK is better than QPSK and 16 QAM.

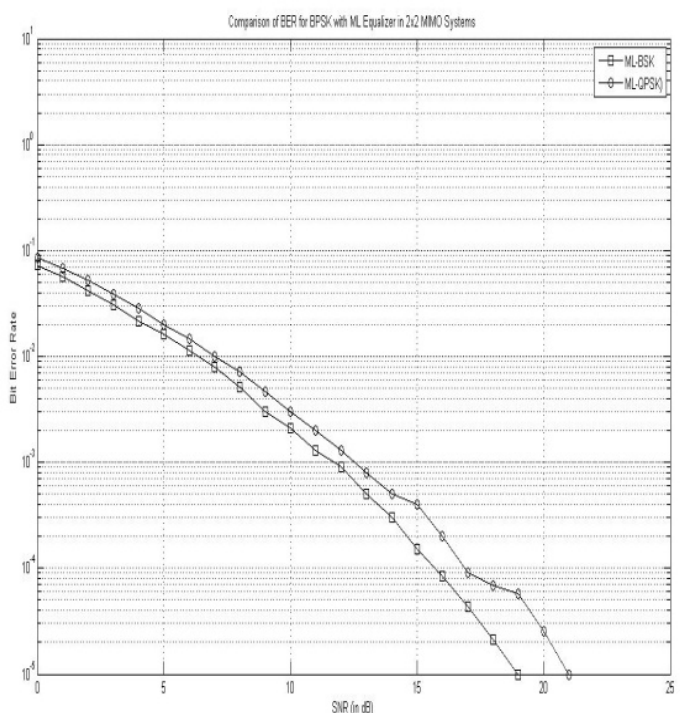

Fig. 2(a)

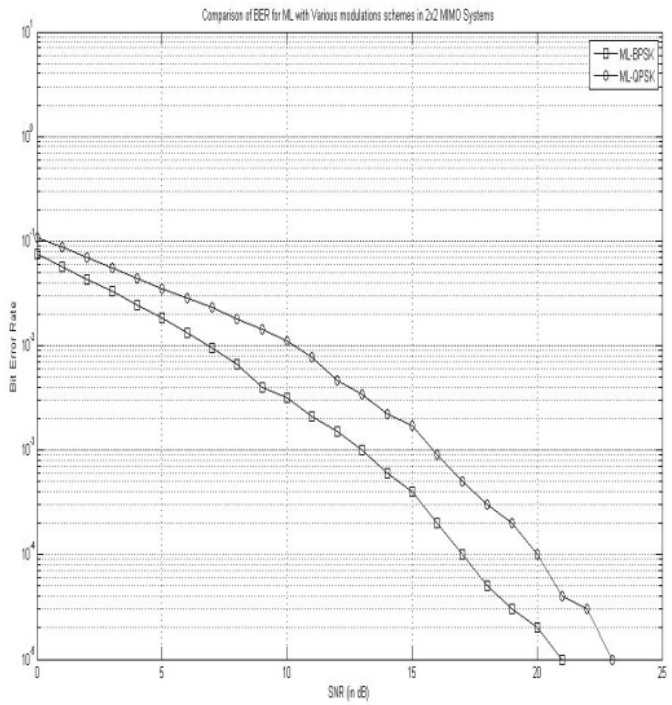

Fig. 2(b) 


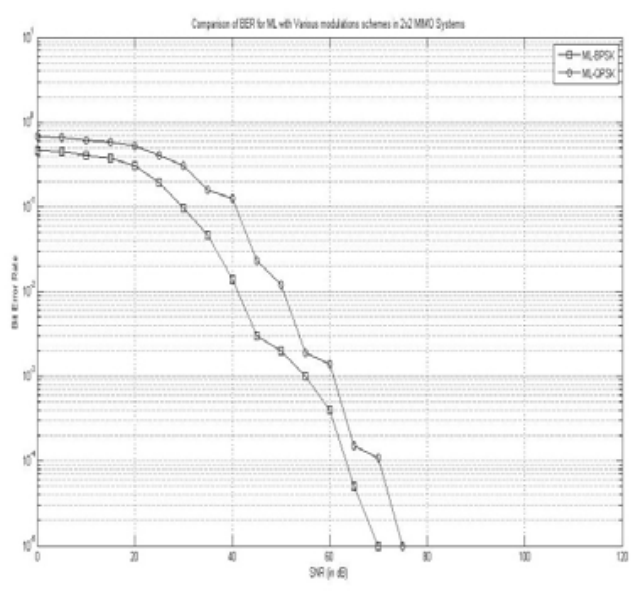

Fig. 2(c)

"Fig.2"shows the comparison of BER for different modulations with ML equalizer.

In figure.2 (a) we have observed that the BER performance of $\mathrm{ML}$ is better than previous discussed equalizers. It is also observed that the complexity of ML equalizer increases with as we go to BPSK to QPSK and with the BPSK modulation we get the best result in comparison to QPSK in Rayleigh Flat Fading Channel, So the order of performance in decreasing order is ML- BPSK $>$ ML - QPSK. In case of Rican Flat Fading Channel, we analyze from Figure 2 (b), the simulated values for BER at 5 $\mathrm{dB}$ SNR are, for BPSK 0.0185, for QPSK 0.0349. So it has been cleared from the Figure 2 (b) that BPSK modulation gives us better result. So the order of performance in decreasing order is ML- BPSK > ML - QPSK. The performance of $\mathrm{ML}$ equalizer is better in Frequency selective fading channel in comparison to other equalizers as it is clear from the Figure 2 (c) that BER doesn't remain constant for a large SNR as in another case. We can conclude that ML equalizer provides us better result in frequency selective fading. At $50 \mathrm{~dB}$ SNR, BER for BPSK is 0.0020 and BER for QPSK is 0.0120.Thus, BPSK provides better result in comparison to QPSK modulation.

\section{CONCLUSIONS}

By applying the MMSE and ML equalizers to Rayleigh flat fading channel, Rician flat fading channel and Rayleigh Frequency Selective fading channel, the performance of ML is better but if we look at the complexity term, the complexity of ML decoder goes on increasing while moving to higher modulation schemes. When equalization is done through frequency selective fading channel, then the performance of MMSE is very poor. Simulation results shows that ML equalizer with BPSK gives better performance as compare to QPSK.

\section{REFERENCES}

[1] Simon, M. K. and Alouini, M. Digital Communication over Fading Channels, John Wiley \& Sons, 2004.

[2] G.J. Foschini and M.J. Gans, "On limits of wireless communications in a fading environment when using multiple antennas", Wireless Personal Communications, vol. 6, pp. 311-335, 1998.

[3] Wang and G. B. Giannakis, "A simple and general parameterization quantifying performance in fading channels," IEEE Transactions Communication., vol. 51, no. 8, pp. 1389-1398, 2003.

[4] Ezio Biglieri, Robert Calderbank, Anthony Constantinides, Andrea Goldsmith, Arogyaswami Paulraj, H. Vincent Poor "MIMO Wireless Communications", Cambridge University Press, 2007.

[5] D. Shiu, P. J. Smith, D. Gesbert, M. Shafi and A. Nayguib, "From theory to practice: An overview of MIMO space-time coded wireless systems," IEEE J. Select. Areas in communications, vol., 21, no. 3, pp. 281-302, 2003.

[6] R. U. Nabar, A. J. Paulraj, D. A. Gore and H. Bolcskei, "An overview of MIMO communications - a key to gigabit wireless," Proceedings of the IEEE, vol. 92, no. 2, pp. 198-218, 2004.

[7] G. D. Golden , G. J. Foschini, R. A. Valenzuela and P. W. Wolniansky, "Detection algorithm and initial laboratory results using v-blast space-time communication architecture, "IEEE Electronic Letters, Vol.35, No.1, pp.14 16, January 1999.

[8] D. Gesbert "MIMO space-time coded wireless systems," Presentation available at http://www.tele.ntnu.no/projects/beats/course.htm. Sept. 2003.

[9] K. Lo, S. Marinkovic, Z C hen and B. Vucetic, "BER performance comparison of layered space time codes", New York, USA ICC 2002.

[10] H. Jafarkhani "Space-time coding: Theory \& Practice", Cambridge University Press, 2005.

[11] I. E. Telatar "Capacity of multi-antenna Gaussian channels, "European Transactions on Telecommunications, vol. 10, no.6, pp.585-595, 1999.

[12] David Tse and Pramod Viswanath," Fundamentals of Wireless Communication Cambridge University Press, 2005.

[13] Rohit Gupta, Amit Grover," BER Performance Analysis of MIMO Systems Using Equalization Techniques", Innovative System Design and Engineering, vol.3, no.10, pp. 11-25, 2012.

\section{AUTHOR'S BIOGRAPHY}

Amit Grover became a Member (M) of Association ISTE in 2006, a Senior Member (SM) of society SELCOME in September 2009, and a Project-In charge (PI) in august 2011 and in September 2012. The author place of birth is Ferozepur, Punjab, India on $27^{\text {th }}$, September 1980.The author received his M. Tech degree in Electronics and Communication Engineering from Punjab Technical University, Kapurthla, Punjab,

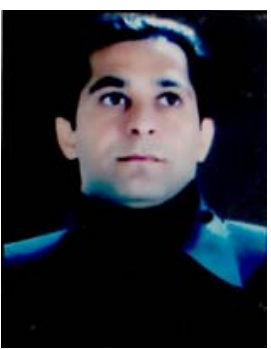
India in 2008 and received his B. Tech degree in Electronics and Communication Engineering from Punjab Technical University, Kapurthala, Punjab, India in 2001. Currently, he is working as an Assistant Professor in Shaheed Bhagat Singh State Technical Campus, Ferozepur, Punjab, India. The author is a Reviewer of many Reputed International Journals. His area of interest includes signal processing, MIMO systems, Wireless mobile communication; high speed digital communications, 4G Wireless Communications and VLSI Design. 